\title{
Response of adult mouse uterus to early disruption of estrogen receptor- $\alpha$ signaling is influenced by Krüppel-like factor 9
}

\author{
C D Simmons, J M P Pabona, Z Zeng, M C Velarde, D Gaddy, F A Simmen and R C M Simmen ${ }^{\mathbf{1}}$ \\ Department of Physiology and Biophysics, University of Arkansas for Medical Sciences, 4301 W Markham Street, Little Rock, Arkansas 72202 , USA \\ ${ }^{1}$ Arkansas Children's Nutrition Center, 15 Children's Way, Little Rock, Arkansas 72202, USA \\ (Correspondence should be addressed to R C M Simmen; Email: simmenrosalia@uams.edu)
}

(M C Velarde is now at the Buck Institute for Age Research, Novato, California 94945, USA)

\begin{abstract}
Inappropriate early exposure of the hormone-responsive uterus to estrogenic compounds is associated with increased risk for adult reproductive diseases including endometrial cancers. While the dysregulation of estrogen receptor- $\alpha$ (ESR1) signaling is well acknowledged to mediate early events in tumor initiation, mechanisms contributing to sustained ESR 1 activity later in life and leading to induction of oncogenic pathways remain poorly understood. We had shown previously that the transcription factor Krüppel-like factor 9 (KLF9) represses ESR1 expression and activity in Ishikawa endometrial glandular epithelial cells. We hypothesized that KLF9 functions as a tumor suppressor, and that loss of its expression enhances ESR 1 signaling. Here, we evaluated the contribution of KLF9 to early perturbations in uterine ESR1 signaling pathways elicited by the administration of synthetic estrogen diethylstilbestrol (DES) to wild-type (WT) and Klf9 null (KO) mice on postnatal days
\end{abstract}

(PNDs) 1-5. Uterine tissues collected at PND84 were subjected to histological, immunological, and molecular analyses. Compared with WT mice, KO mice demonstrated larger endometrial glands and lower endometrial gland numbers; DES exposure exacerbated these differences. Loss of KLF9 expression resulted in increased glandular ESR1 immunoreactivity with DES, without effects on serum estradiol levels. Quantitative RT-PCR analyses indicated altered expression of uterine genes commonly dysregulated in endometrial cancers (Akt1, Mmp9, Slpi, and Tgfß1) and of those involved in growth regulation (Fos, Myc, Tert, and $S y k$ ), with loss of Klf9, alone or in concert with DES. Our data support a molecular network between KLF9 and ESR1 in the uterus, and suggest that silencing of KLF9 may contribute to endometrial dysfunctions initiated by aberrant estrogen action.

Journal of Endocrinology (2010) 205, 147-157

\section{Introduction}

The steroid hormone $17 \beta$-estradiol $\left(\mathrm{E}_{2}\right)$ regulates numerous physiological and developmental processes in target tissues by binding to its cognate receptors estrogen receptor- $\alpha$ (ESR1) and $-\beta$ (ESR2) in the presence of numerous co-regulators (Heldring et al. 2007). In the uterus, ESR 1 is the predominant isoform, and its presence is necessary for reproductive functions (Couse \& Korach 1999, Matthews \& Gustafsson 2003). Indeed, mice null for Esr1 are infertile in contrast to Esr2 KO mice (Hewitt \& Korach 2003) due in part to the failure of the uteri to develop properly (Couse et al. 1995). Conversely, the unopposed activity of uterine ESR1 promotes the development and progression of endometrial cancers in humans and animal models (Di Cristofano \& Ellenson 2007). Multiple mechanisms underlie the control of ESR1 expression and activity; these include regulation at the levels of transcription involving multiple promoters (Kos et al. 2001); alternative splicing (Heldring et al. 2007); posttranslational modifications via phosphorylation, acetylation, and polyubiquitination (Fu et al. 2004, Masuyama \& Hiramatsu 2004, Atsriku et al. 2009); and posttranscriptional silencing by micro-RNA targeting (Castellano et al. 2009). The aforementioned complex regulation of ESR1 occurring at many levels predicts that perturbations in any or all of these mechanisms will lead to reproductive dysfunctions and diseases.

Endocrine-disrupting chemicals (EDCs) mimic or antagonize the effects of naturally occurring substances that elicit physiological responses in target cells (Newbold et al. 2006, 2007). Numerous studies are aimed at elucidating the mechanism(s) by which such chemicals exert potentially devastating health consequences in subjects upon environmental exposure (Diamanti-Kandarakis et al. 2009). Diethylstilbestrol (DES), a synthetic estrogen, earned its notoriety as an EDC based on the documented increased breast cancer risk in exposed mothers (Greenberg et al. 1984, Colton et al. 1993, Palmer et al. 2006) and vaginal clear cell adenocarcinoma in daughters exposed in utero (Herbst et al. 1971). In mice, the decreased fecundity and higher incidence 
of structural abnormalities of the female reproductive tract with prenatal exposure to DES (Newbold 1995, Kaufman et al. 2000) were recapitulated by postnatal DES administration (postnatal days (PNDs) 1-5), a critical window of reproductive tract differentiation (Newbold et al. 1990). The majority of the uterine defects observed with DES had been attributed to its ability to alter the expression, co-regulatory protein recruitment, and downstream signaling events of ESR1 (Newbold 1995, Couse et al. 2001, Yamashita 2004). Indeed, the overexpression of ESR1 enhanced the progression of DES-induced uterine adenocarcinoma (Couse et al. 1997). While these findings implicate perturbations in ESR1 signaling to play a pivotal role in DES-mediated reproductive dysfunctions, the precise mechanisms and participating molecules remain poorly understood.

Krüppel-like factor (KLF) 9, a member of the Sp/KLF family of transcription factors (Suske et al. 2005), is a 244-amino acid protein characterized by three C-terminal zinc finger motifs that are important for binding to GC-rich sequences in DNA (Imataka et al. 1992). By using Klf9 null mice and endometrial cell lines with KLF9 knockdown in vitro, our laboratory has shown that KLF9 is a progesterone receptor (PGR) co-regulator (Zhang et al. 2002a, 2003), and depending on cell context, a negative (Velarde et al. 2007) or positive (Pabona et al. 2009) regulator of ESR1 signaling. In mice, Klf9 null mutation was associated with uterine hypoplasia and reduced uterine progesterone sensitivity, leading to a subfertility phenotype (Simmen et al. 2004), and with delayed parturition due to dysregulated PGR-A expression (Zeng et al. 2008). Klf9 mRNA expression was recently shown to be attenuated in abnormal uterine conditions such as in a mouse model of endometriosis and in human leiomyoma (Rackow \& Taylor 2008, Lee et al. 2009). These findings indicate that Klf9 plays an important role with ESR1 and PGR in uterine growth, function, and pathology.

In the present study, we evaluated the existence of a uterine ESR1/KLF9 crosstalk in the context of perturbed ESR1 signaling elicited by postnatal (days 1-5) DES exposure of wild-type (WT) and Klf9 null mice. We have shown that relative to those of WT mice, adult uteri of Klf9 null mice had reduced luminal epithelial (LE) height, larger endometrial glands, and lower endometrial gland numbers, and that the DES effects on these indices were dependent on Klf9 expression. We have further shown that uterine Klf 9 expression temporally lagged behind ESR 1 expression (Yamashita et al. 1989, Sato et al. 1992, Newbold 1995), and was insensitive to early DES exposure. We have demonstrated that while loss of Klf9 expression had no effect on basal ESR1 expression in endometrial stromal and glandular epithelial compartments of adult uteri, early DES-exposed Klf9 null mutants exhibited higher glandular ESR1 expression. Finally, we found altered expression of a subset of genes frequently dysregulated in endometrial cancers (Akt1, Mmp9, and Slpi) and of pro-proliferative genes (Fos, Myc, Tert, and Syk), with loss of Klf9 alone or in concert with DES exposure. These findings provide support for KLF9's participation in endometrial ESR1-sensitive developmental events, and suggest that silencing of KLF9 may contribute to the etiology of endometrial cancers initiated by aberrant ESR1 signaling.

\section{Materials and Methods}

\section{Animals and treatments}

Animal studies were conducted in compliance with protocols approved by the Institutional Animal Care and Use Committee of the University of Arkansas for Medical Sciences. C57BL/6J Klf9 WT and mutant mice were propagated as published previously (Morita et al. 2003, Simmen et al. 2004). To eliminate confounding effects of maternal phenotype on pup viability (Simmen et al. 2004), WT and mutant offspring were generated from male Klf9 $(+/-) \times$ female Klf9 $(+/-)$ matings. At birth (PND1) until PND5, female pups were injected daily with $2 \mu \mathrm{g}$ of DES dissolved in sesame oil (vehicle) or sesame oil alone (Oil; control) (Newbold et al. 1990). At PND21, the pups were weaned and genotyped. For purposes of this study, only WT and $\mathrm{KO}(-/-)$ mice were compared. At PND84, body weight and stage of estrus (by vaginal smears) were determined, and the females were killed by $\mathrm{CO}_{2}$ asphyxiation. For each mouse, the ovary pairs and uterine horns were weighed. One uterine horn was placed in 10\% neutral buffered formalin and processed for histomorphometry, while the other horn was processed for RNA isolation. A total of 16 WT (9 and 7 respectively for Oil and DES) and $16 \mathrm{KO}(8$ and 8 respectively for Oil and DES) mice were used for tissue collection and analyses (Fig. 1).

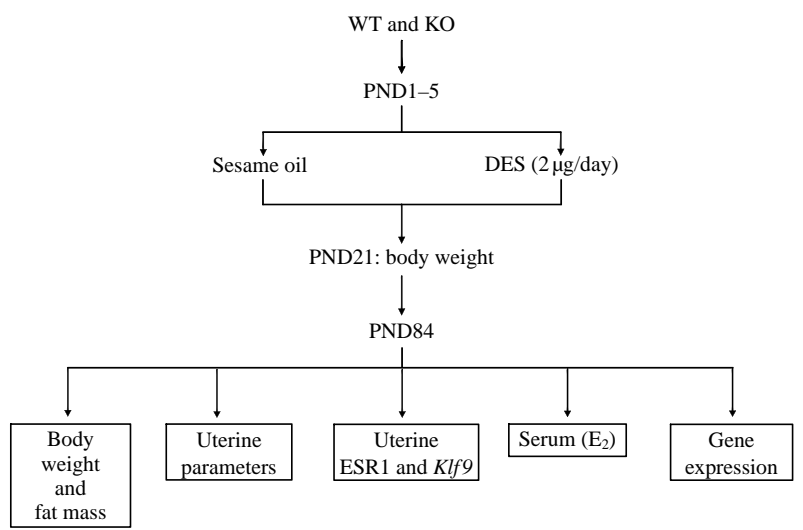

Figure 1 Experimental design using KIf9 WT and null mutant mice according to the DES model of endometrial carcinoma. PND, postnatal day. 
RNA isolation and quantitative RT-PCR

Total RNA was isolated from uterine tissues with TRIzol reagent (Invitrogen) following the manufacturer's protocol. RNA concentrations were determined using the Nanodrop ND-1000 spectrophotometer (Nanodrop, Wilmington, DE, USA). cDNA was synthesized from $1 \mu \mathrm{g}$ of total RNA using the Taqman Reverse Transcription Reagents kit (Applied Biosystems, Foster City, CA, USA), and analyzed by quantitative RT-PCR (QPCR) using SYBR Green (Bio-Rad) and the Bio-Rad MyiQ optical module and iCycler thermal cycler as described previously (Velarde et al. 2006). Intron-flanking primers were designed to eliminate the amplification of genomic DNA using the Primer Express software (Applied Biosystems), and were obtained from Integrated DNA Technologies, Inc. (Coralville, IA, USA). The primer sequences (sense and antisense respectively) and the corresponding PCR product sizes are given in Table 1. A standard curve was generated by serially diluting pooled cDNAs from all animals beginning with the most concentrated cDNA pool designated as 10000 arbitrary units. Focused QPCR array (Mouse Cancer PathwayFinder, SABiosciences, Frederick, MD, USA) analyses followed protocols described by the manufacturer, using cDNAs prepared from pooled RNAs (of same amount) isolated from DES-treated WT $(n=5)$ and Klf9 mutant $(n=4)$ mice.

Immunohistochemistry, uterine morphometry, and X-Gal staining

Whole uteri were fixed in 10\% neutral buffered formalin as described previously (Simmen et al. 2004). Sections (5 $\mu \mathrm{m})$ were mounted on poly(lysine)-coated slides (Fisher Scientific, Pittsburgh, PA, USA), and used for uterine morphometry or immunohistochemistry. For morphometrical analyses, the sections were deparaffinized in xylene, rehydrated in a series of alcohols, and stained with hematoxylin and eosin following standard protocols. LE height and gland numbers were quantified using the Carl Zeiss Axiovert 200M microscope equipped with a Zeiss Axiocam HRc camera and Axiovision software (Carl Zeiss, Inc., Thornwood, NY, USA). Measurements were taken from five distinct fields $(100 \times)$ per section, and data from 2 to 3 serial sections from each Klf9 WT and $\mathrm{KO}$ mouse $(n=3$ mice per treatment per genotype) were analyzed. For detection and quantification of ESR1-immunostaining cells, sections were treated with 3\% hydrogen peroxide for $30 \mathrm{~min}$ to quench endogenous peroxidase activity and with Citra Plus (Biogenex, San Ramon, CA, USA) in a microwave set at power 10 for $105 \mathrm{~s}$ and at power 1 for $10 \mathrm{~min}$ to unmask antigen. Sections were incubated in a blocking solution (VectaStain ABC kits; Vector Laboratories, Burlingame, CA, USA) overnight to reduce non-specific staining prior to incubation for 16-24 h with rabbit anti-mouse polyclonal ESR1 antibody (1:250 dilution; sc-542, Santa Cruz Biotechnology, Santa Cruz, CA, USA) in a humidity chamber at $4{ }^{\circ} \mathrm{C}$. Incubation with biotinylated anti-rabbit secondary antibody (1:200 dilution; VectaStain ABC kits) was carried out for $30 \mathrm{~min}$ at room temperature. Sections were stained with DAB Chromogen (3,3'-diaminobenzidine, Dako, Carpinteria, CA, USA), counterstained with hematoxylin, dehydrated, cleared, and coverslipped for examination under a microscope. Immunostained cells were counted from four randomly chosen fields (200X magnification) per slide per mouse $(n=3-4$ mice per genotype per treatment). KLF9 expression was quantified by X-Gal (5-bromo-4-chloro-3-indolyl- $\beta$-D-galactopyranoside) staining because Klf9 mutant mice were generated by knock-in of the $L a c Z$ gene into the open reading frame of Klf9 exon 1 (Morita et al. 2003), and because in-house and commercially available anti-KLF9 antibodies give high background by immunohistochemistry. Tissue sections from Klf9 heterozygous $(+/-)$ mice were prepared and processed for X-Gal staining as described previously (Simmen et al. 2004, Zeng et al. 2008).

Table 1 Mouse primer sequences used for quantitative RT-PCR

\begin{tabular}{|c|c|c|c|}
\hline & Sense & Antisense & Product size $(b p)$ \\
\hline \multicolumn{4}{|l|}{ Gene } \\
\hline Rn18s & 5'-TGCTCTTAGCTGAGTGTCCCG-3' & 5'-TCATGGCCTCAGTTCCGAAA-3' & 153 \\
\hline Gapdh & 5'-TGTGTCCGTCGTGGATCTGA-3' & 5'-GCATCGAAGGTGGAAGAGTGG-3' & 173 \\
\hline Klf13 & 5'-ACACAGGTGAGAGGCCTTTCG-3' & 5'-AGCATGCCTGGGTGGAAGT-3' & 199 \\
\hline Slpi & 5'-AAGTCCTGCGGCCTTTTACCT-3' & 5'-GGCATTGTGGCTTCTCAAGCT-3' & 154 \\
\hline Pten & $5^{\prime}$-CCCACCACAGCTAGAACTTATCAA-3' & $5^{\prime}$-TCCGTCCCTTTCCAGCTTTAC-3' & 110 \\
\hline Myc & $5^{\prime}$-TACTTGGAGGAGACATGGTGAATC- $3^{\prime}$ & 5'-AGAAGCCGCTCCACATACAGTC- $3^{\prime}$ & 102 \\
\hline Akt1 & 5'-GACTGACACCAGGTATTTCGATGA-3' & $5^{\prime}$-СТCCGСТСАСТGTCСАCACA-3' & 102 \\
\hline FOS & 5'-TGAAGAGGAAGAGAAACGGAGAAT- $3^{\prime}$ & $5^{\prime}$-TCCGCTTGGAGTGTATCTGTCA-3' & 102 \\
\hline Syk & 5'-AGGGTCAAAGACCAATGGAAAA-3' & 5'-CTGTCAATGCGGTAGTGCAATACT-3' & 108 \\
\hline Tert & 5'-CCTCCAGACAGTCTGCATCAATAT-3' & 5'-TCTTCСТAACACGCTGGTCAAA-3' & 104 \\
\hline Tgfb1 & 5'-GCCTGAGTGGCTGTCTTTTGAC-3' & $5^{\prime}$-CACAAGAGCAGTGAGCGCTG-3' & 101 \\
\hline Mmp9 & 5'-TCGCGTGGATAAGGAGTTCTC-3' & 5'-AACTCACACGCCAGAAGAATTTG-3' & 107 \\
\hline
\end{tabular}




\section{Western blots}

Western blot analyses for KLF9 protein levels in Oil (control)and DES-treated WT mouse uteri were performed as described previously (Zhang et al. 2002a). Protein extracts were prepared from TRIzol reagent (Invitrogen) following the manufacturer's protocol. The protein extracts were dissolved in 1\% SDS, diluted 1:10 in water, and quantified using the BCA Protein Assay Kit (Fisher Scientific), with BSA diluted in $0 \cdot 1 \%$ SDS as the standard. Blots were probed with rabbit anti-KLF9 antibody (1:1000; generated in-house; Zhang et al. 2002a), followed by HRP-conjugated goat anti-rabbit IgG (1:1500; Bio-Rad) secondary antibody. Expression was detected with Amersham ECL Plus reagent (GE Healthcare, Piscataway, NJ, USA), and band intensities were quantified using Quantity One 1-D Analysis Software (version 4.6.5; Bio-Rad).

\section{$R I A$}

Approximately $500 \mu \mathrm{l}$ of whole blood were collected by closed cardiac puncture from PND84 female Klf9 WT and KO mice. Serum was separated from whole blood by centrifugation at $4600 \mathrm{~g}$ for $1 \mathrm{~h}$, and was stored at $-20^{\circ} \mathrm{C}$ prior to analysis. Serum $\mathrm{E}_{2}$ levels were measured using the Ultrasensitive Estradiol kit (Diagnostic Systems Laboratories, Webster, TX, USA) following the manufacturer's protocol.

\section{Data analysis}

Data (mean \pm s.E.M.) were analyzed for statistical significance by two-way ANOVA followed by all pairwise multiple comparison procedures (Tukey's test). Where applicable, Student's $t$-test was performed to determine the effect of treatment within a particular genotype. All statistical analyses were performed using the SigmaStat software (Systat Software, Chicago, IL, USA) version 3.5. Differences were considered significant at $P<0 \cdot 05$.

\section{Results}

\section{DES-associated growth effects with Klf9 mutation}

WT and KO mice administered either Oil (vehicle) or DES daily beginning at birth for 5 days (PND1-5) were examined for body weights at weaning (PND21) and at PND84. At PND21, KO mice were numerically lighter than WT mice, although the difference did not reach statistical significance. With DES treatment, WT mice were significantly lighter, while $\mathrm{KO}$ mice were unaffected, relative to respective Oil-treated controls (Fig. 2A). At PND84, WT mice were heavier with DES administration (Fig. 2B), and this was accompanied by a numerical (albeit not significant) increase in gonadal fat pad weight (Fig. 2C). Klf9 mutant mice showed no differences in body or fat pad weights with DES relative to the corresponding Oil group.

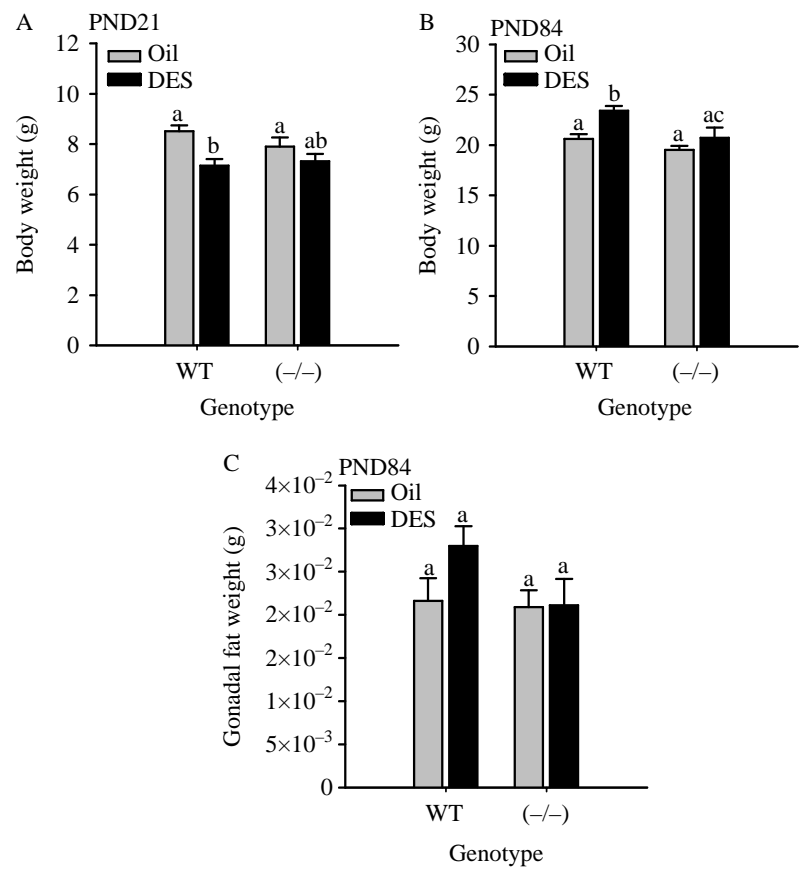

Figure 2 Body and gonadal fat pad weights of control (Oil) and DES-treated Klf9 WT and null mutant mice at postnatal days (PNDs) 21 (A) and 84 (B and C). Data are mean \pm s.E.M. Means with different superscripts differed at $P<0 \cdot 05$ by two-way ANOVA followed by Tukey's test.

\section{DES-associated alterations in uterine parameters with Klf9 mutation}

We had shown previously that KLF9 negatively regulates ESR1 signaling in endometrial glandular epithelial cells (Velarde et al. 2007). To investigate DES effects on the uterus with loss of Klf9 expression, we evaluated a number of uterine parameters (wet weight, LE height, gland size, and numbers), which are well-recognized indicators of dysfunctional estrogenic stimulation, in PND84 WT and KO mice. Uterine wet weights relative to body weight did not differ with genotype in control and DES treatment groups, with both WT and $\mathrm{KO}$ mice showing reduced uterine weights from corresponding Oil control with DES treatment (Fig. 3A). LE height varied as a function of Klf9 mutation, with Oiltreated KO mice showing reduced LE heights than WT mice, comparable to those of DES-treated WT mice (Fig. 3B). Uterine gland numbers were decreased with loss of Klf9, and further reductions were observed for both genotypes with DES treatment (Fig. 3C). Loss of Klf9 expression was associated with a significant increase in uterine gland size (Fig. 3D), and while both genotypes showed increased gland areas with DES treatment, the significant differences between WT and KO mice were maintained (Fig. 3D). Serum $\mathrm{E}_{2}$ levels did not differ with Klf9 status among the Oil-treated groups, and DES suppressed $\mathrm{E}_{2}$ levels only for WT mice (Fig. 3E). Ovarian weights normalized to body weight were unaffected by genotype or treatment (Fig. 3F). 

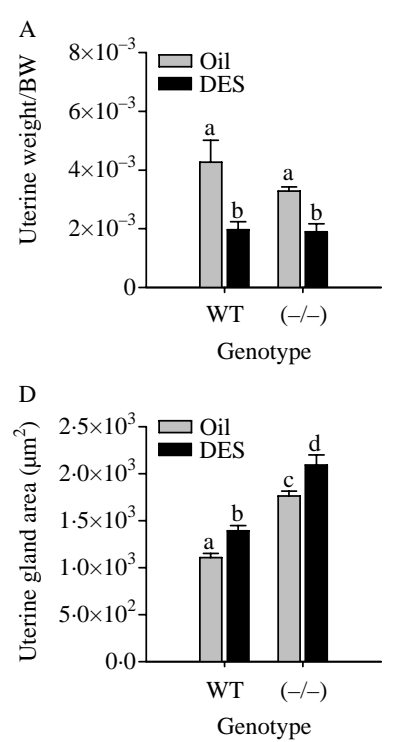
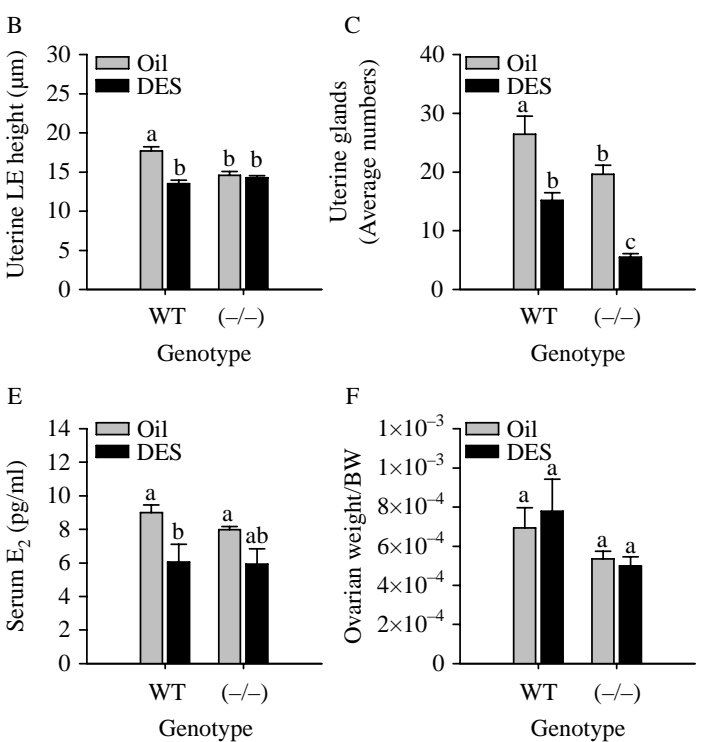

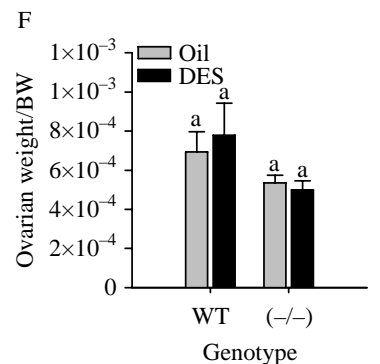

Figure 3 Uterine and ovarian indices of control (Oil) and DES-treated KIf9 WT and null mutant mice at PND84. Data are mean \pm s.E.M. Means with different superscripts differed at $P<0 \cdot 05$ by two-way ANOVA followed by Tukey's test.

\section{Developmental expression of uterine KLF9}

Disruption of molecular and structural parameters in the mouse uterus with early DES exposure was lost with ESR1 null mutation, suggesting DES dysregulation of ESR1dependent events as an underlying cause (Couse et al. 2001). Moreover, our previous studies suggested the existence of KLF9/ESR1 crosstalk under distinct cellular contexts (Velarde et al. 2007, Pabona et al. 2009). We determined whether KLF9 expression was manifested in the uterus during the period of DES exposure to evaluate the possibility of KLF9 contributing to later uterine phenotype with early perturbations in ESR1 signaling by DES. The temporal expression of $\mathrm{Klf} 9$ gene was evaluated in uterine cryosections of Klf9 heterozygous mice at PND5, 11, 21, and 28 by X-Gal staining (Fig. 4A). Uterine Klf9 expression was undetectable at PND5 and PND11, and was highly visible at PND21 and PND28 (Fig. 4A). Consistent with previous findings (Simmen et al. 2004), Klf9 gene was expressed predominantly in endometrial stroma, sporadically in glands, and was undetectable in luminal epithelium. QPCR (Fig. 4B) and western blot (Fig. 4C) analyses showed robust expression of both KLF9 mRNA and protein in uteri of PND84 WT mice, which were unaffected by early DES treatment.

\section{KLF9 regulation of ESR 1 expression with DES treatment}

Because the effects of DES, which signals through ESR1, on estrogen-responsive uterine parameters (e.g. LE height, gland number, and size) differed with Klf9 genotype at PND84 (Fig. 3), the expression of uterine ESR1 as a function of KLF9 expression status and DES treatment was assessed by immunohistochemistry (Fig. 5A). Immunoreactive ESR1 protein was detectable in all endometrial cellular compartments. Notably, glands showed the highest staining, with most cells in each gland showing strong nuclear immunoreactivity. The numbers of nuclear stromal ESR1 (quantified as percentage of ESR1-immunopositive cells relative to the total cells counted) were similar in Oil-treated WT and $\mathrm{KO}$ mice, with a significant increase noted for WT mice but not for KO mice with early DES exposure (Fig. 5B). In the glandular compartment, nuclear ESR1-immunopositive cells were significantly higher only in DES-treated KO mice; Oil- and DES-treated WT and control KO mice showed comparable numbers of nuclear ESR1immunopositive cells (Fig. 5B).

\section{Altered uterine gene expression in WT and Klf9 $\mathrm{KO}$ mice with DES treatment}

To further evaluate the contribution of KLF9 to ESR1 signaling, we next analyzed the expression of genes previously shown to be regulated by KLF9 (Simmen et al. 2004) and/or DES treatment (Hong et al. 2006, Newbold et al. 2007) in the mouse uterus. KLF13, a member of the Sp/KLF family of transcription factors, exhibits the highest similarity in primary structure to KLF9 (Kaczynski et al. 2003). We had shown previously that Klf13 expression is increased in uteri of Klf9 null mice (Simmen et al. 2004). PTEN is a tumor suppressor whose loss of expression is associated with the development of endometrial carcinoma in mice (Daikoku et al. 2008), and is considered a prognostic marker for the disease in humans (Lian et al. 2006). Secretory leukocyte protease inhibitor 

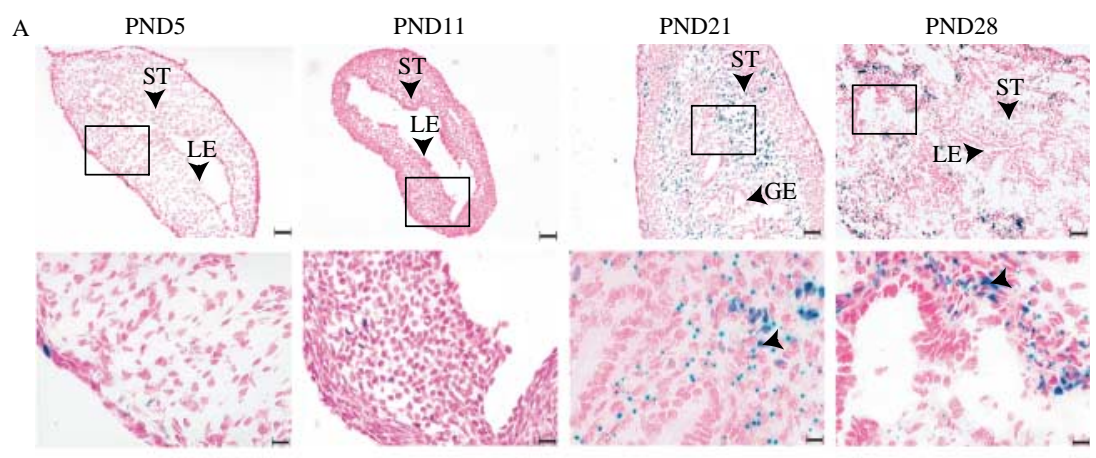

B
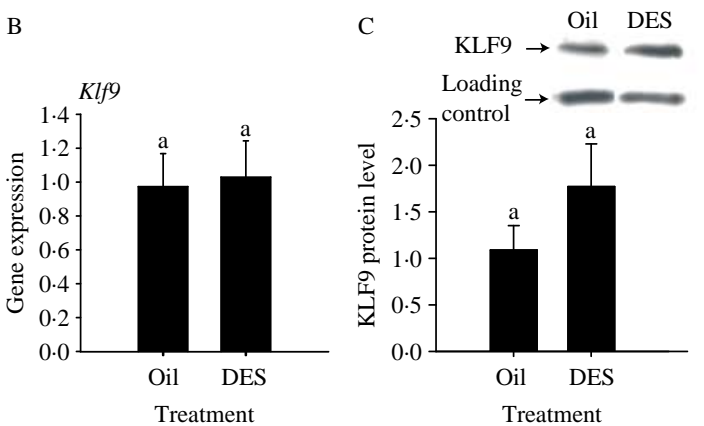

Figure 4 Temporal expression of uterine Klf9. (A) Uterine X-Gal staining demonstrating the absence of Klf9 gene expression at PND5 and PND11 and predominantly stromal Klf9 expression (arrow) at PND21 and PND28, using Klf9 (+/-) mice. Images were taken at $100 \times$ (upper panels; scale bar, $40 \mu \mathrm{m}$ ) and $400 \times$ (lower panels; scale bar, $10 \mu \mathrm{m}$ ).

(B) Uterine Klf9 mRNA expression was quantified by QPCR and normalized to $18 \mathrm{~S}$ mRNA for Oil- and DES-treated KIf9 WT mice at PND84. Data (mean \pm s.E.M.) are expressed as fold change relative to WT Oil treatment group ( $n=4-8$ mice per treatment per genotype). (C) Western blot analyses for KLF9 protein levels in Oil- and DES-treated Klf9 WT mice at PND84. Data (mean \pm s.E.M.; $n=2$ independent analyses) are expressed relative to loading control, a non-specific $(<17 \mathrm{kDa})$ protein detected in the same blot.

(SLPI) is a glandular epithelial protein whose increased expression is linked to a highly proliferative uterine state (Zhang et al. 2002b) and Klf9 expression (Simmen et al. 2008). In control (Oil-treated) mice, Klf13 mRNA expression was elevated significantly with Klf9 mutation (Fig. 6A), and DES treatment did not alter this pattern of expression with genotype. Pten mRNA expression was numerically reduced with loss of Klf9 expression in the Oil groups, albeit the decrease was not statistically significant (Fig. 6B). DES treatment decreased Pten expression $(P=0 \cdot 047)$ in WT mice, and maintained the low levels of Pten in KO mice (Fig. 6B). By contrast, uterine Slpi mRNA expression, while comparable between Oil-treated WT and Klf9 null mice, was increased by DES exposure but only in Klf9 null mice $(P=0 \cdot 028$; Fig. 6C).

To expand the repertoire of genes whose expression may be further disrupted by Klf9 mutation in the background of early DES exposure, we performed additional studies using the Mouse Cancer PathwayFinder PCR Array. Analyses of the uterine genes altered between DES-treated WT and Klf9 mutant mice (from the 84 genes representing six biological pathways involved in transformation and tumorigenesis and present on the array) identified a total of 23 genes whose expression was either upregulated or downregulated by at least twofold (data not shown). In order to validate the results from the microarrays, a subset of these upregulated genes were examined for differential expression by QPCR using cDNAs prepared from uterine RNAs of Oil- and DES-treated WT and $\mathrm{KO}$ mice (Fig. 7A). Interestingly, with the exception of Fos, whose transcript levels were only altered with DES in KO mice, the majority of the genes (Akt1, Myc, Tert, and SYk) demonstrated expression patterns that were increased with loss of Klf 9 expression, independent of DES treatment. Mmp 9 and Tgfb1 showed comparable expression patterns, with higher levels in Klf9 KO mice relative to WT mice and which tended to decrease with early DES exposure for KO mice but not for WT mice (Fig. 7A).

\section{Early effects of KLF9 mutations on gene expression}

The gene expression data from the above analyses (Figs 6 and 7A) identified Akt1, Fos, and Slpi as genes that are differentially regulated by early DES exposure in the background of Klf9 null mutation at PND84. To evaluate 

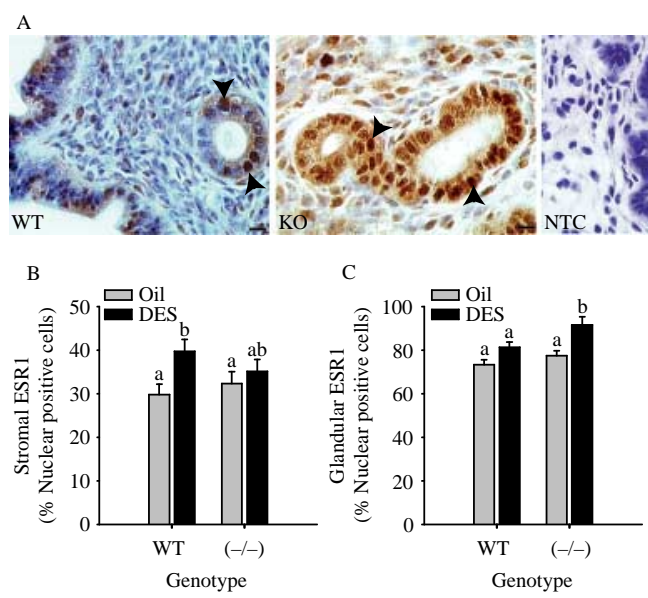

Figure 5 Endometrial stromal and glandular epithelial ESR1 expression levels as a function of Klf9 genotype and DES exposure. (A) Representative images of ESR1 immunostaining in stromal and glandular epithelial cells of Oil-treated PND84 Klf9 WT and KO mice. Images are at $400 \times$ magnification; scale bar, $10 \mu \mathrm{m}$. Control (NTC) represents tissue section from Klf9 mutant mice in the absence of primary antibody. Arrows indicate nuclear staining. PND84 stromal (B) and glandular epithelial (C) ESR1 quantification (mean \pm S.E.M.) for $n=3-4$ mice per genotype per treatment (Oil or DES). Means with different superscripts differed at $P<0 \cdot 05$ by two-way ANOVA followed by Tukey's test.

whether these genes may function as early markers of disrupted ESR1 signaling influenced by loss of Klf9 expression, their expression levels were evaluated in uteri of prepubertal (PND28) WT and Klf9 null mice. As shown in Fig. 7B, only the Slpi expression pattern duplicated that found at PND84. While Akt1 transcript levels were affected by Klf9 null mutation similar to that at PND84 $(P=0.059$ for WT Oil versus KO Oil), $A k t 1$ response to DES exposure at PND28 was different from that at PND84. Fos expression at PND28 remained unchanged with Klf9 genotype and DES treatment, which contrasted with the findings for PND84 uteri (Fig. 7A).

In silico analysis of selected genes in endocarcinoma

To correlate the changes in gene expression identified in the present study with previously reported alterations in gene expression for endometrial tumors (Mutter et al. 2001), an online cancer data-mining resource (www.oncomine.org) was used as described previously (Simmen et al. 2010). Table 2 lists those genes that were commonly altered between the findings reported here and the published dataset (Mutter et al. 2001). The changes in expression of KLF9, AKT1, MMP9, and SLPI, but not of TGFB1, were in the same direction for endometrial tumors as that found in uterine tissues of Klf9 null mice relative to WT mice. By contrast, the uterine expression of MYC, SYK, and TERT genes, which were altered in Klf9 null mice relative to WT mice, did not differ in endometrial tumors from corresponding non-diseased uteri (Mutter et al. 2001).

\section{Discussion}

In this study, we established a central role for KLF9 in the response of uterine endometrial cells to early perturbations in ESR1 signaling. Using the well-characterized DES-induced model for dysregulation of ESR1 function leading to pathology (Newbold et al. 1990, 2007), we showed that loss of Klf9 expression in the absence of DES altered uterine morphological parameters (LE height, gland numbers, and gland size) comparable to those for DES-treated WT mice, and increased endometrial gland sensitivity to DES. We demonstrated that Klf9 null mutation enhanced glandular ESR1 expression with DES, and this was positively associated with increased expression of Slpi, a gene encoding an endometrial gland-secreted protein whose overexpression is implicated in the pathogenesis of many cancers, including that of the endometrium, due to its pro-proliferative actions (Zhang et al. 2002b, Chen et al. 2004). Finally, we demonstrated that unlike ESR1, whose uterine expression was found to be an early target of DES, occurring within days of DES administration (Sato et al. 1992) and which remained dysregulated later in life, KLF9 expression was undetectable in the developing uterus (PND5 and PND11) and was unaffected by DES later in life. Given that endometrial
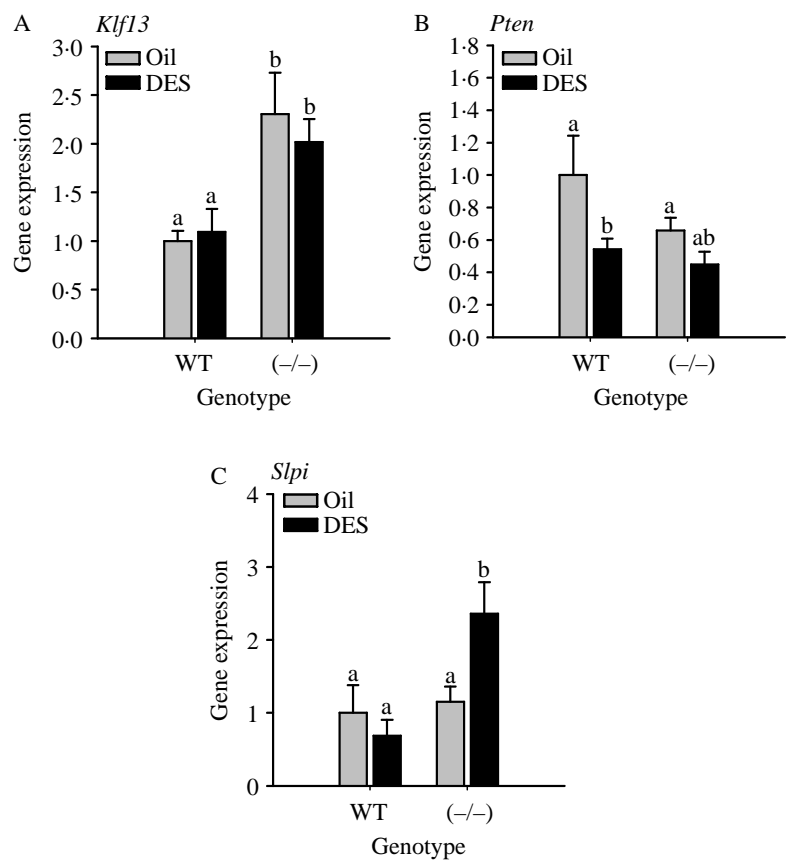

Figure 6 Uterine expression of selected genes as a function of $K$ lf9 genotype and DES exposure. mRNA levels were quantified by QPCR and normalized to GAPDH (for Klf13) or 18S mRNA (for Pten and SIpi) for Oil- and DES-treated Klf9 WT and null mutant mice at PND84. Data (mean \pm S.E.M.) are expressed as fold change relative to WT Oil treatment group ( $n=4-8$ mice per treatment per genotype). Means with different superscripts differed at $P<0.05$ by two-way ANOVA followed by Tukey's test. 

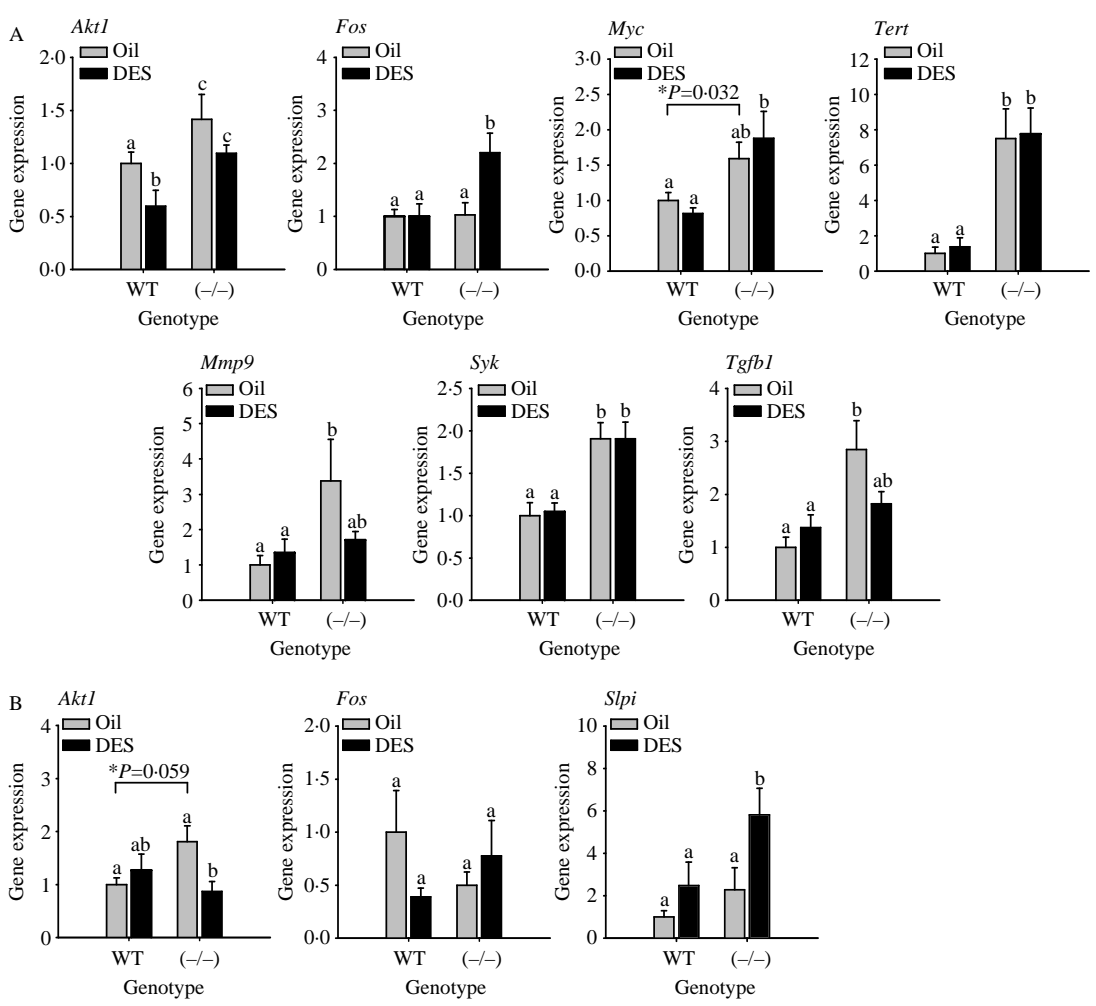

Figure 7 Differential expression of uterine genes identified from Focused QPCR Array. Uterine mRNA expression at PND84 (A) and PND28 (B) was quantified by QPCR and normalized to $18 \mathrm{~S}$ mRNA for Oil- and DES-treated Klf9 WT and null mice. Data (mean \pm S.E.M.) are expressed as fold change relative to WT Oil treatment group ( $n=4-8$ mice per treatment per genotype). Means with different superscripts differed at $P<0 \cdot 05$ by two-way ANOVA followed by Tukey's test. For Myc (A) and Akt1 (B), additional analyses by Student's $t$-test between bracketed groups indicated significance difference (asterisk).

cancer originates from endometrial glands (Di Cristofano \& Ellenson 2007) and consistent with our previous report of increased ESR 1 expression with $\mathrm{E}_{2}$ treatment upon siKLF9 knockdown in Ishikawa (glandular epithelial) cells (Velarde et al. 2007), our results suggest that KLF9 constitutes an important regulator of ESR 1 signaling in endometrial glands, and that loss of this regulation may predispose to increased risk for endometrial cancers.

Our findings that early (postnatal) DES exposure alters adult uterine phenotype (PND84) are consistent with previously reported long-term consequences of DES-induced early developmental perturbations for this tissue in mice (Couse et al. 2001). The precise mechanism by which early disruption of ESR 1 signaling leads to abnormalities in uterine function of adults remains poorly understood. Epigenetic changes involving modifications to chromatin structure due to aberrant histone methylation, histone acetylation, and DNA methylation are increasingly considered to underlie the onset and progression of cancers (Ellis et al. 2009). Consistent with this, neonatal DES exposure has been reported to alter genomic DNA methylation due to changes in the expression of uterine DNA methyltransferases (Sato et al. 2009). Our data showing the lack of co-expression of KLF9 with ESR1 in uteri of mice in the first two weeks of life (Yamashita et al. 1989, Sato et al. 1992, Newbold 1995) suggest that KLF9 mediates glandular ESR 1 expression in adult uteri subsequent to and/or independent of early events arising from epigenetic modifications of ESR1. KLF9 may alter nuclear ESR1

Table 2 A subset of differentially expressed genes in human endocarcinoma associated with $K L F 9$ expression status ${ }^{\text {a }}$

\section{Endocarcinoma ${ }^{\mathrm{b}}$}

$\boldsymbol{P}_{\text {value }}{ }^{\mathrm{c}}$

Gene

AKT1

KLF9

MMP9

SLPI

TGFB1

$M Y C$

$S Y K$

TERT

$\begin{array}{ll}+ & 0 \cdot 00018 \\ - & 0 \cdot 035 \\ + & 0 \cdot 00019 \\ + & 0 \cdot 08 \\ - & 0 \cdot 014 \\ \text { NC } & 0 \cdot 408 \\ \text { NC } & 0 \cdot 548 \\ \text { NC } & 0.904\end{array}$

an silico analysis of Mutter et al. (2001).

$\mathrm{b}(+)$, increased with carcinoma; $(-)$, decreased with carcinoma; $\mathrm{NC}$, no change with carcinoma.

${ }^{\mathrm{c}} P$ value relative to normal endometrium. 
expression through effects on ESR1 stability, nuclear localization, and/or promoter activity in the adult uteri; the latter is consistent with our previous findings that reduced KLF9 expression prevented the recruitment of ESR1 to its own promoter (Velarde et al. 2007) to elicit ESR1-negative autoregulation. Alternatively or in addition, given that KLF9 functionally interacts with PGR-B (Zhang et al. 2003), the loss of PGR-B/KLF9 interaction in KO mouse uteri may have resulted in loss of PGR antagonism of ESR 1 expression, thereby leading to sustained ESR 1 signaling. We found that PGR-B transcript levels in PND84 mouse uteri were not altered as a function of genotype and early DES exposure in this study (data not shown), consistent with KLF9 effects at the level of PGR transactivity. While our data do not address the stage in uterine development wherein loss of Klf9 expression on ESR1 signaling is manifested, we suggest that this probably occurs as early as PND28 based on the comparable patterns of uterine Slpi expression in PND28 and PND84 DES-exposed Klf9 null mice when compared with the corresponding aged WT mice, and given that Slpi is an ESR1-induced gene (Chen et al. 2004). Further studies will address ESR1 expression in Klf9 null uteri of younger (prepubertal) mice. Taken together, our data suggest that while the contribution of epigenetic mechanisms to dysregulated ESR1 expression with DES exposure (Li et al. 2003, Bromer et al. 2009) cannot be excluded, KLF9 involvement in the regulation of ESR 1 and Slpi expression is probably a consequence of its action at the level of the ESR1 promoter, albeit this may not involve direct physical interactions between KLF9 and ESR1 (Velarde et al. 2007).

To determine if changes in estrogen-responsive parameters were attributable to altered ovarian function, we measured serum $\mathrm{E}_{2}$ levels and ovarian tissue weights of adult WT and Klf9 null mice with and without early DES exposure. The comparable $\mathrm{E}_{2}$ levels in both genotypes as well as the lack of significant differences in ovarian wet weights as a function of genotype and DES administration predict a similar lack of effects on serum $P$ levels, consistent with our previous reports of the absence of major ovarian outcomes with Klf9 null mutation (Simmen et al. 2004, Zeng et al. 2008). Thus, ESR1/KLF9-dependent effects noted in glandular epithelial cells are largely ovarian independent, and are probably a consequence of direct uterine targeting by KLF9.

We used a combination of candidate gene and focused gene array approaches to identify genes whose expression was differentially altered in adult (PND84) WT and Klf9 mutant uteri, based on their relationships to KLF9, ESR1, and/or cancer risk. Of the uterine genes confirmed to be regulated with loss of Klf9 expression, two major categories are proposed: category 1 includes genes that are regulated by KLF9, independent of ESR1 (DES) signaling (Klf13, Myc, Tert, $S Y k$, and Akt1), while category 2 includes genes that are influenced by KLF9/ESR 1 cross-regulation (Fos and Slpi). The increased expression of pro-proliferative/cell survival, ESR1-regulated genes Akt1 (Altomare \& Testa 2005, Vilgelm et al. 2006), Myc (Cole 1986), Syk (Coopman et al. 2000), and Tert (Boggess et al. 2006) with loss of Klf9 expression is consistent with the potential tumor suppressor function of KLF9, as suggested by the reported loss of KLF9 expression in endometrial tumors (Mutter et al. 2001, Simmen et al. 2008). Moreover, the coincident elevated expression of $M y c$ and Tert mRNAs is consistent with the observation by Wu et al. (1999) that MYC upregulates Tert promoter activity. Fos and Slpi are estrogen-regulated genes, whose gene products exhibit pro-proliferative activities (Zhang et al. 2002b, Li et al. 2003, Chen et al. 2004); hence, their increased expression with Klf9 mutation coincident with increased ESR1 expression (with DES exposure) suggests a pathway linking the loss of KLF9-mediated ESR1-negative regulation to enhanced proliferation, and provides further support to potential KLF9 tumor suppressor activity. The increased expression of Klf13 with Klf9 null mutation implicates a regulatory loop between these highly related family members, as suggested from our previous studies (Simmen et al. 2004). Pten mutations are among the earliest identifiable aberrations in endometrial carcinoma (Lian et al. 2006); hence, our findings of a robust decrease in Pten transcript levels in DESexposed WT uteri relative to control WT uteri are consistent with increased incidence of endometrial carcinoma in perinatal DES-exposed mice (Newbold et al. 2006, 2007). While we demonstrated only a trend for decreased uterine Pten expression in Klf9 null mutants without and with DES exposure relative to WT mice, Pten transcript levels of Klf9 null mutants without DES exposure were numerically comparable to those of DES-exposed WT mice, suggesting that dysregulation of Pten expression by early DES exposure is partially mimicked by Klf9 null mutation. In this regard, recent data showing Slpi upregulation in the uteri of Pten heterozygous mice which develop atypical hyperplasias and carcinomas in situ (Lian et al. 2006), and that conditional deletion of endometrial Pten resulted in the rapid development of endometrial carcinoma in mice (Daikoku et al. 2008) are consistent with linkages between the expression of Klf9, Slpi, and Pten and uterine tumorigenesis, as inferred from the present results. Studies ongoing in our laboratory to evaluate uterine tumor development in Klf9 null mice with and without postnatal DES exposure will be critical to address the link between KLF9 and uterine endocarcinoma.

In summary, data presented herein suggest a novel role for the transcription factor KLF9 in modulating uterine responses to ESR1-dependent DES actions, the latter leading to a dysfunctional uterus. Our in vivo model superimposing Klf9 null mutation to early (postnatal) DES exposure has revealed a molecular network between KLF9 and ESR 1 that is operative in the uterus involving key estrogen-regulated genes that may constitute early predictors of uterine tumor development, and may also underlie infertility associated with EDC exposure. The direct evidence for KLF9 as a tumor suppressor awaits further studies; nonetheless, our results raise the intriguing possibility that the silencing of KLF9 by either genetic and/or epigenetic mechanisms, as demonstrated for many tumor 
suppressors and oncogenes (Di Cristofano \& Ellenson 2007), may contribute to increased susceptibility for developing endometrial cancers initiated by aberrant estrogen action.

\section{Declaration of interest}

The authors declare that there is no conflict of interest that could be perceived as prejudicing the impartiality of the research reported.

\section{Funding}

This work was supported by the National Institutes of Health grant HD21961 (to RCMS and FAS) and a grant from the Arkansas Biosciences Institute (to RCMS and DG). CS was a recipient of an NIH Supplement Award (HD21961-19S \& -20S); the Arkansas Children's Hospital Research Institute Student and Clinical Staff Research Intramural Grant, and the University of Arkansas for Medical Sciences Committee for Allocating Graduate Student Research Funds Award.

\section{Acknowledgements}

We would like to thank members of our laboratories for helpful discussions during the course of this study, and Dr K Shankar, R Till and A Greenway for technical assistance.

\section{References}

Altomare DA \& Testa JR 2005 Perturbations of the AKT signaling pathway in human cancer. Oncogene 24 7455-7464.

Atsriku C, Britton DJ, Held JM, Schilling B, Scott GK, Gibson BW, Benz CC \& Baldwin MA 2009 Systematic mapping of posttranslational modifications in human estrogen receptor-alpha with emphasis on novel phosphorylation sites. Molecular and Cellular Proteomics 8 467-480.

Boggess JF, Zhou C, Bee-Jump VL, Gehrig PA \& Whang YE 2006 Estrogen receptor-dependent regulation of telomerase activity in human endometrial cancer cell lines. Gynecologic Oncology 103 417-424.

Bromer JG, Wu J, Zhou Y \& Taylor HS 2009 Hypermethylation of homeobox A10 by in utero diethylstilbestrol exposure: an epigenetic mechanism for altered developmental programming. Endocrinology $\mathbf{1 5 0}$ 3376-3382.

Castellano L, Giamas G, Jacob J, Coombes RC, Lucchesi W, Thiruchelvam P, Barton G, Jiao LR, Wait R, Waxman J et al. 2009 The estrogen receptor-alpha-induced microRNA signature regulates itself and its transcriptional response. PNAS 106 15732-15737.

Chen D, Xu X, Cheon YP, Bagchi MK \& Bagchi IC 2004 Estrogen induces expression of secretory leukocyte protease inhibitor in rat uterus. Biology of Reproduction 71 508-514.

Cole MD 1986 The myc oncogene: its role in transformation and differentiation. Annual Review of Genetics 20 361-384.

Colton T, Greenberg ER, Noller K, Resseguie L, Van Bennekom C, Heeren T \& Zhang Y 1993 Breast cancer in mothers prescribed diethylstilbestrol in pregnancy. Journal of the American Medical Association 269 2096-2100.

Coopman PJ, Do MT, Barth M, Bowden ET, Hayes AJ, Basyuk E, Blancato JK, Vezza PR, McLeskey SW, Mangeat PH et al. 2000 The Syk tyrosine kinase suppresses malignant growth of human breast cancer cells. Nature 406 742-747.

Couse JF \& Korach KS 1999 Estrogen receptor null mice: what have we learned and where will they lead us? Endocrine Reviews 20 358-417.
Couse JF, Curtis SW, Washburn TF, Eddy EM, Schomberg DW \& Korach KS 1995 Disruption of the mouse oestrogen receptor gene: resulting phenotypes and experimental findings. Biochemical Society Transactions $\mathbf{2 3}$ 929-935.

Couse JF, Davis VL, Hanson RB, Jefferson WN, McLachlan JA, Bullock BC, Newbold RR \& Korach KS 1997 Accelerated onset of uterine tumors in transgenic mice with aberrant expression of the estrogen receptor after neonatal exposure to diethylstilbestrol. Molecular Carcinogenesis 19 236-242.

Couse JF, Dixon D, Yates M, Moore AB, Ma L, Maas R \& Korach KS 2001 Estrogen receptor- $\alpha$ knockout mice exhibit resistance to the developmental effects of neonatal diethylstilbestrol exposure on the female reproductive tract. Developmental Biology 238 224-238.

Daikoku T, Hirota Y, Tranguch S, Joshi AR, DeMayo FJ, Lydon JP, Ellenson LH \& Dey SK 2008 Conditional loss of uterine Pten unfailingly and rapidly induces endometrial cancer in mice. Cancer Research 68 5619-5627.

Diamanti-Kandarakis E, Bourguignon JP, Giudice LC, Hauser R, Prins GS, Soto AM, Zoeller RT \& Gore AC 2009 Endocrine-disrupting chemicals: an Endocrine Society scientific statement. Endocrine Reviews 30 293-342.

Di Cristofano A \& Ellenson LH 2007 Endometrial carcinoma. Annual Review of Pathology 2 57-85.

Ellis L, Atadja PW \& Johnstone RW 2009 Epigenetics in cancer: targeting chromatin modifications. Molecular Cancer Therapentics 8 1409-1420.

Fu M, Wang C, Zhang X \& Pestell RG 2004 Acetylation of nuclear receptors in cellular growth and apoptosis. Biochemical Pharmacology 68 1199-1208.

Greenberg ER, Barnes AB, Resseguie L, Barrett JA, Burnside S, Lanza LL, Neff RK, Stevens M, Young RH \& Colton T 1984 Breast cancer in mothers given diethylstilbestrol in pregnancy. New England Journal of Medicine 311 1393-1398.

Heldring N, Pike A, Andersson S, Matthews J, Cheng G, Hartman J, Tujague M, Ström A, Treuter E, Warner M et al. 2007 Estrogen receptors: how do they signal and what are their targets. Physiological Reviews 87 905-931.

Herbst AL, Ulfelder H, Poskanzer DC \& Longo LD 1971 Adenocarcinoma of the vagina. Association of maternal stilbestrol therapy with tumor appearance in young women. American Journal of Obstetrics and Gynecology 181 1574-1575.

Hewitt SC \& Korach KS 2003 Oestrogen receptor knockout mice: roles for oestrogen receptor alpha and beta in reproductive tissues. Reproduction 125 143-149.

Hong E, Park S, Choi K, Leung P \& Jeung E 2006 Identification of estrogen-regulated genes by microarray analysis of the uterus of immature rats exposed to endocrine disrupting chemicals. Reproductive Biology and Endocrinology 449.

Imataka H, Sogawa K, Yasumoto K, Kikuchi K, Sasano K, Kobayashi A, Hayami M \& Fujii-Kuriyama Y 1992 Two regulatory proteins that bind to the basic transcription element (BTE). A GC box sequence in the promoter region of the rat P-4501A1 gene. EMBO Journal 11 3663-3671.

Kaczynski J, Cook T \& Urrutia R 2003 Sp1- and Krüppel-like transcription factors. Genome Biology 4206.

Kaufman RH, Adam E, Hatch EE, Noller K, Herbst AL, Palmer JR \& Hoover RN 2000 Continued follow-up of pregnancy outcomes in diethylstilbestrol-exposed offspring. Obstetrics and Gynecology 96 483-489.

Kos M, Reid G, Denger S \& Gannon F 2001 Minireview: genomic organization of the human ERalpha gene promoter region. Molecular Endocrinology 15 2057-2063.

Lee B, Hongling D \& Taylor HS 2009 Experimental murine endometriosis induces DNA methylation and altered gene expression in eutopic endometrium. Biology of Reproduction 80 79-85.

Li S, Hansman R, Newbold R, Davis B, McLachlan JA \& Barrett JC 2003 Neonatal diethylstilbestrol exposure induces persistent elevation of $\mathrm{c}$-fos expression and hypomethylation in its exon- 4 in mouse uterus. Molecular Carcinogenesis 38 78-84.

Lian Z, De Luca P \& Di Cristofano A 2006 Gene expression analysis reveals a signature of estrogen receptor activation upon loss of Pten in a mouse model of endometrial cancer. Journal of Cellular Physiology 208 255-266.

Masuyama H \& Hiramatsu Y 2004 Involvement of suppressor for Gal 1 in the ubiquitin/proteasome-mediated degradation of estrogen receptors. Journal of Biological Chemistry 279 12020-12026. 
Matthews J \& Gustafsson JA 2003 Estrogen signaling: a subtle balance between ER alpha and ER beta. Molecular Interventions 3 281-292.

Morita M, Kobayashi A, Yamashita T, Shimanuki T, Nakajima O, Takahasi S, Ikegami S, Inokuchi K, Yamashita K \& Yamamoto M 2003 Functional analysis of basic transcription element binding protein by gene targeting technology. Molecular and Cellular Biology 23 2489-2500.

Mutter GL, Baak JPA, Fitzgerald JT, Gray R, Neuberg D, Kust G, Gentleman R, Gullans S, Wei L \& Wilcox M 2001 Global expression changes of constitutive and hormonally regulated genes during endometrial neoplastic transformation. Gynecologic Oncology 83 177-185.

Newbold RR 1995 Cellular and molecular effects of developmental exposure to diethylstilbestrol: implications for other environmental estrogens. Environmental Health Perspectives 103 83-87.

Newbold RR, Bullock BC \& McLachlan JA 1990 Uterine adenocarcinoma in mice following developmental treatment with estrogens: a model for hormonal carcinogenesis. Cancer Research 50 7677-7681.

Newbold RR, Padilla-Banks E \& Jefferson WN 2006 Adverse effects of the model environmental estrogen diethylstilbestrol are transmitted to subsequent generations. Endocrinology 147 S11-S17.

Newbold RR, Jefferson WN, Grissom SF, Padilla-Banks E, Snyder RJ \& Lobenhofer EJ 2007 Developmental exposure to diethylstilbestrol alters uterine gene expression that may be associated with uterine neoplasia later in life. Molecular Carcinogenesis 46 783-796.

Pabona JMP, Velarde MC, Zeng Z, Simmen FA \& Simmen RCM 2009 Nuclear receptor co-regulator Krüppel-like factor 9 and prohibitin 2 expression in estrogen-induced epithelial cell proliferation in the mouse uterus. Journal of Endocrinology 200 63-73.

Palmer JR, Wise LA, Hatch EE, Troisi R, Titus-Ernstoff L, Strohsnitter W, Kaufman R, Herbst AL, Noller KL, Hyer M et al. 2006 Prenatal diethylstilbestrol exposure and risk of breast cancer. Cancer Epidemiology, Biomarkers \& Prevention 15 1509-1514.

Rackow BW \& Taylor HS 2008 Submucosal uterine leiomyomas have a global effect on molecular determinants of endometrial receptivity. Fertility and Sterility. DOI: 10.1016/j.fertnstert.2008.03.029

Sato T, Okamura H, Ohta Y, Hayashi S, Takamatsu Y, Takasugi N \& Iguchi T 1992 Estrogen receptor expression in the genital tract of female mice treated neonatally with diethylstilbestrol. In Vivo 6 151-156.

Sato K, Fukata H, Kogo Y, Ohgane J, Shiota K \& Mori C 2009 Neonatal exposure to diethylstilbestrol alters expression of DNA methyltransferases and methylation of genomic DNA in the mouse uterus. Endocrine Journal 56 $131-139$.

Simmen RCM, Eason RR, McQuown JR, Linz AL, Kang T-J, Chatman L, Till SR, Fujii-Kuriyama Y, Simmen FA \& Oh SP 2004 Subfertility, uterine hypoplasia, and partial progesterone resistance in mice lacking the Krüppel-like factor $9 /$ basic transcription element-binding protein-1 (Bteb1) gene. Journal of Biological Chemistry 279 29286-29294.

Simmen FA, Su Y, Xiao R, Zeng Z \& Simmen RC 2008 The Krüppel-like factor 9 (KLF9) network in HEC-1-A endometrial carcinoma cells suggests the carcinogenic potential of dys-regulated KLF9 expression. Reproductive Biology and Endocrinology 641.

Simmen R, Pabona J, Velarde M, Simmons C, Rahal O \& Simmen F 2010 The emerging role of Kruppel-like factors in endocrine-responsive cancers of female reproductive tissues. Journal of Endocrinology 204 223-231.
Suske G, Bruford E \& Philipsen S 2005 Mammalian SP/KLF transcription factors: bring in the family. Genomics 85 551-556.

Velarde MC, Iruthayanathan M, Eason RR, Zhang D, Simmen FA \& Simmen RCM 2006 Progesterone receptor transactivation of the secretory leukocyte protease inhibitor gene in Ishikawa endometrial epithelial cells involves recruitment of Krüppel-like factor 9/basic transcription element binding protein-1. Endocrinology 147 1969-1978.

Velarde MC, Zeng Z, McQuon JR, Simmen FA \& Simmen RCM 2007 Krüppel-like factor 9 is a negative regulator of ligand-dependent estrogen receptor alpha signaling in Ishikawa endometrial adenocarcinoma cells. Molecular Endocrinology 21 2988-3001.

Vilgelm A, Lian Z, Wang H, Beauparlant S, Klein-Szanto A, Ellenson LH \& Di Cristofano Antonio 2006 Akt-mediated phosphorylation and activation of estrogen receptor alpha is required for endometrial neoplastic transformation in Pten + / - mice. Cancer Research 66 3375-3380.

Wu KJ, Grandori C, Amacker M, Simon-Vermot N, Polack A, Lingner J \& Dalla-Favera R 1999 Direct activation of TERT transcription by c-MYC. Nature Genetics 21 220-224.

Yamashita S 2004 Ontogenic expression of estrogen receptor coactivators in the reproductive tract of female mice neonatally exposed to diethylstilbestrol. Reproductive Toxicology 18 275-284.

Yamashita S, Newbold RR, McLachlan JA \& Korach KS 1989 Developmental pattern of estrogen receptor expression in female mouse genital tracts. Endocrinology 125 2888-2896.

Zeng Z, Velarde MC, Simmen FA \& Simmen RCM 2008 Delayed parturition and altered myometrial progesterone receptor isoform A expression in mice null for Krüppel-like factor 9. Biology of Reproduction $\mathbf{7 8}$ 1029-1037.

Zhang D, Zhang X-L, Michel FJ, Blum JL, Simmen FA \& Simmen RCM 2002a Direct interaction of the Krüppel-like family (KLF) member, BTEB1 and progesterone receptor mediates progesteroneresponsive gene expression in endometrial epithelial cells. Endocrinology $14162-73$.

Zhang D, Simmen RCM, Michel FJ, Zhao G, Vale-Cruz D \& Simmen FA $2002 b$ Secretory leukocyte protease inhibitor mediates proliferation of human endometrial epithelial cells by positive and negative regulation of growth-associated genes. Journal of Biological Chemistry 277 29999-30009.

Zhang XL, Zhang D, Michel FJ, Blum JL, Simmen FA \& Simmen RCM 2003 Selective interactions of Kruppel-like factor 9/basic transcription element-binding protein with progesterone receptor isoforms A and B determine transcriptional activity of progesterone-responsive genes in endometrial epithelial cells. Journal of Biological Chemistry 278 21474-21482.

Received in final form 9 February 2010

Accepted 17 February 2010

Made available online as an Accepted Preprint 17 February 2010 